\title{
Nal and HI 3-D density distribution in the solar neighbourhood ${ }^{\star}$
}

\author{
J.-L. Vergely ${ }^{1}$, R. Freire Ferrero ${ }^{1}$, A. Siebert ${ }^{1}$, and B. Valette ${ }^{2}$ \\ 1 Observatoire Astronomique de Strasbourg, UMR 7550, Université Louis Pasteur, Strasbourg, France \\ 2 LGIT-Savoie, Université de Savoie, 73376 Le-Bourget-du-Lac, France
}

Received 10 December 1999 / Accepted 13 November 2000

\begin{abstract}
A study of the local interstellar medium (LISM) using a robust inversion method, similar to current tomography techniques, is applied to compiled data on neutral interstellar absorbers and Hipparcos parallaxes. We present here the 3-D distribution of two neutral species, NaI and HI. Our analysis enables us to obtain a reliable 3-D density distribution of the IS matter in the solar neighbourhood, providing a new basis for the discussion of origin, present state and evolution of the LISM. We show that neutral IS matter is distributed in compact clouds or in cloud complexes with cavities between them. It is now easy to distinguish the so-called Local Bubble and the Loop I cavities and also two tunnels linking the Local Bubble to the outer regions of the galaxy, away from the galactic plane. Better accuracy is achieved for NaI, as to a larger number of lines-of-sight and target stars than are available for HI. A rather detailed NaI 3-D density distribution is obtained with a 40 pc smoothing length. The extended high-density regions in the $\mathrm{NaI}$ and $\mathrm{HI}$ maps are correlated which is not the case for the diffuse regions. The density ratio $\rho_{\mathrm{HI}} / \rho_{\mathrm{NaI}}$ is lower or equal to $9.1 .10^{8} \pm 3.10^{8}$ for extended high density clouds.
\end{abstract}

Key words. ISM: bubbles - ISM: clouds - Galaxy: solar neighbourhood - stars: distances - methods: statistical

\section{Introduction}

Analysis of IS data samples made by different authors has allowed the approximate characterization of the distribution of matter in the local interstellar medium (LISM) (Frisch 1995) and, with a minor degree of confidence, the farther ISM.

In this way, it has been determined that the Sun is embedded in a relatively empty cavity, known as the Local Bubble, and that there are other neighbouring cavities (Cox \& Reynolds 1987) delimited by several dense clouds.

Phenomena such as supernova explosions or hot star winds shape the ISM (Cox \& Reynolds 1987), forming low density regions, which occur in contrast with highdensity and relatively compact regions such as molecular dust clouds.

The Local Bubble is filled with hot gas, either ionized by residual EUV radiation from a recent supernova event $(t<300000 \mathrm{yr})$ (Vallerga \& Welsh 1995) or resulting from heat conduction at the interface of the cold Local Cloud and the hot Local Bubble (Slavin 1989).

The ionisation state of the different elements in the ISM provides information about physical conditions pre-

\footnotetext{
Send offprint requests to: J.-L. Vergely, e-mail: vergely@astro.u-strasbg.fr

* Using observations from the ESA Hipparcos space astrometry mission.
}

vailing therein: neutral species like $\mathrm{NaI}$ trace low temperature regions; ionized species, are more probably associated with high temperature regions.

In this work, we present the 3-D density distribution of $\mathrm{NaI}$ and $\mathrm{HI}$ in the vicinity of the Sun, using recently compiled data on IS HI and NaI (Garcia 1991; Welsh et al. 1994; Sfeir et al. 1999).

These data were obtained on equivalent widths and column densities, obtained either from equivalent width of IS lines and a theoretical curve of growth or from spectral line fitting.

Column densities describe the fluctuations of the local density of matter (see the following section). The transition from column density $\left(\mathrm{cm}^{-2}\right)$ to local density $\left(\mathrm{cm}^{-3}\right)$ is not trivial and requires inverse techniques. Such techniques were used for the first time in the spatial distribution of dust clouds (Vergely 1998; Vergely et al. 1997; Chen et al. 1998; Valette \& Vergely, in preparation) and are here applied to column densities of neutral species.

The most recent IS NaI cartographies give the isocontours of the column densities or equivalent widths in order to define the limit of the Local Bubble and the position of the NaI clouds (Welsh et al. 1998; Sfeir et al. 1999). Here we apply an explicit inverse scheme, since tomography methods provide a qualitative and quantitative description of the 3-D distribution of IS matter in 
the galactic neighbourhood. The main advantage of such methods is the use of all the information in a coherent way for the different directions, enabling the determination of the local density of matter. The solution obtained by the inversion techniques is global, i.e. it considers the medium as a whole object. This is made possible because of the underlying physical 3-D density correlation.

The distance of the target stars gives us the depth of the column density. A set of different depths and column densities in a line-of-sight gives information regarding the fluctuations of density along this line-of-sight.

The accuracy of such an estimation of density depends on the accurate estimation of the stellar distances. The Hipparcos catalogue (Perryman et al. 1997) gives very accurate parallaxes $\left(\sigma_{\pi} \simeq 1\right.$ mas) independent of the stellar type in a sphere of $200 \mathrm{pc}$ radius.

In the following section we present a brief description of the inversion technique. We describe the process leading to a uniform data set and present the $3-\mathrm{D}$ density distribution of the neutral species, HI and NaI, together with an estimation of their accuracy, in a cube with sides of $500 \mathrm{pc}$ centered on the Sun. Finally, the ratio $\rho_{\mathrm{HI}} / \rho_{\mathrm{NaI}}$ is determined from these maps.

\section{Column density and local density}

The observed quantity of IS absorption arises from IS lines which are superimposed over stellar spectra: the equivalent width is a measured quantity from observations, but column density derived.

The column density, $N$, is given as a function of density $\rho$ by:

$N_{\mathrm{NaI}, \mathrm{HI}}=\int_{0}^{R} \rho_{\mathrm{NaI}, \mathrm{HI}}(r) \mathrm{d} r$,

which means that $\rho$ is simply the derivative of the column density:

$\rho_{\mathrm{NaI}, \mathrm{HI}}=\frac{\mathrm{d} N_{\mathrm{NaI}, \mathrm{HI}}}{\mathrm{d} r}$.

Of course, it is impossible to derive the data directly. The inverse method we used comes from statistical techniques that have been applied in geophysical analyses (Tarantola \& Valette 1982). We have adapted this method for astrophysical applications as the inversion of the column density to obtain the spatial density (Vergely 1998; Valette \& Vergely, in preparation).

This method supposes that we have an a priori knowledge of some characteristics of the model which we hope to determine. The inverse process computes the fluctuations produced by the data around the mean a priori model.

It is now accepted that the density of IS matter decreases strongly with the galactic latitude. A convenient description of this behaviour is to suppose a priori an exponential decrease with height above the galactic plane (Vergely 1998):

$\rho_{\text {prior }}(r, b)=\rho_{0} \exp \left(-\frac{|r \sin (b)|}{h_{0}}\right)$.
The value of $h_{0}$ is taken as $100 \mathrm{pc}$, in agreement with the distribution of dust clouds (Chen et al. 1998).

The density is a positive quantity and, in this way, can be parametrised by a log-normal law as follows:

$\rho(x, y, z)=\rho_{0} \exp (\alpha(x, y, z)) \exp \left(-\frac{|z|}{h_{0}}\right)$

where $\alpha$ is the unknown parameter which is assumed to follow a Gaussian statistical law. In the following numerical applications, we have taken $\rho_{0}(\mathrm{HI})=0.5 \mathrm{~cm}^{-3}$ and $\rho_{0}(\mathrm{NaI})=110^{-9} \mathrm{~cm}^{-3}$.

Given the limited number of lines-of-sight, we must assume some regularizing properties for $\alpha$ (recall that $\left.\alpha(x, y, z)=\log \left(\rho(x, y, z) / \rho_{\text {prior }}\right)\right)$. We introduce a smoothing length, $\xi$, which corresponds to the mean distance between neighbouring stars in the sample. This condition is given a priori and can be written as a variance-covariance operator (Tarantola \& Valette 1982) which has the following kernel:

$$
\begin{aligned}
C_{0}\left(x, x^{\prime}, y, y^{\prime}, z, z^{\prime}\right) & =\sigma_{\alpha}(x, y, z) \sigma_{\alpha}\left(x^{\prime}, y^{\prime}, z^{\prime}\right) \\
& \times \exp \left(-\frac{\left(\left(x-x^{\prime}\right)^{2}+\left(y-y^{\prime}\right)^{2}+\left(z-z^{\prime}\right)^{2}\right)}{\xi^{2}}\right) .
\end{aligned}
$$

This kernel is the autocorrelation function between two points of the space: $(x, y, z)$ and $\left(x^{\prime}, y^{\prime}, z^{\prime}\right)$. The parameter $\xi$ is asymptotically related to $\sigma_{\alpha}$ by the relation:

$\sigma_{\alpha} \propto\left(\frac{1}{\xi}\right)^{3 / 2}$

The parameter $\sigma_{\alpha}$ is fixed a priori and controls the acceptable fluctuation level of the model. A large value of $\sigma_{\alpha}$ will induce more contrast in the maps than will a small value. The choice of the two a priori parameters ( $\xi$ and $\left.\sigma_{\alpha}\right)$ is conditioned by the stability of the inversion process and the distance between stars. For example, if we choose a small value of $\xi$, the correlation between nearby lines-of-sight will be neglected and the information will be concentrated along the line-of-sight. On the other hand, if the value of $\xi$ is chosen too large, the small details on the map will disappear and the density fluctuations will become too smoothed. When there are no lines-of-sight, the recovered density will be modeled by the a priori density value.

Finally, we chose a $\chi^{2}$ minimisation criteria in order to control the algorithm convergence:

$$
\chi^{2}=\sum_{i=1, n} \frac{\left(N_{i}(\text { obs })-N_{i}(\text { model })\right)^{2}}{\sigma_{N i}^{2}},
$$

where $N_{i}$ (obs) and $N_{i}$ (model) are, respectively, the observed and the modeled column densities.

The observational data set could contain some outliers. In order to take them into account, we can use an exponential norm algorithm that is more robust than the Gaussian one. In fact, the inverse method adjust data to an a priori model, rejecting data which apparently does not correspond to the a priori general characteristics of the model. 


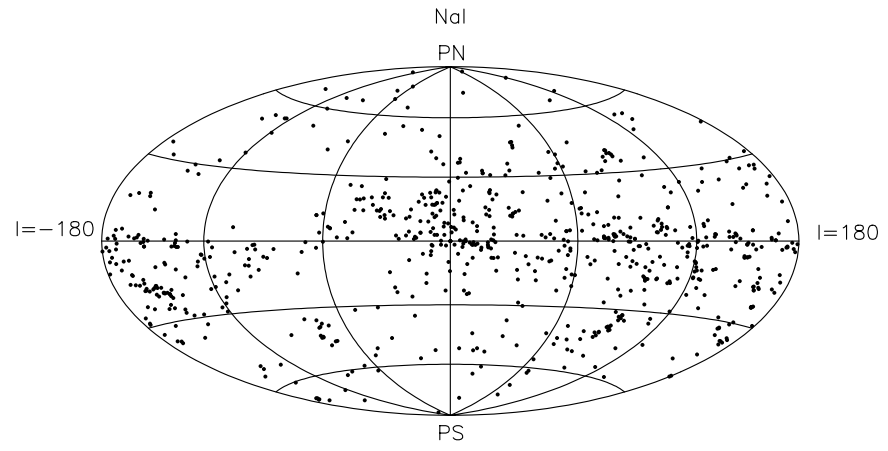

Fig. 1. Projection of the NaI target stars on the celestial sphere for the stars closer than $1.5 \mathrm{kpc}$

At first, the smoothing length $\xi$ was taken equal to $40 \mathrm{pc}$ for the NaI near the Sun, after the analysis of the spatial distribution of targets in the NaI database, the mean distance between them and some trial-and-error experiments.

For the HI, an appropriate correlation length $\xi$ is $60 \mathrm{pc}$, according to the data at our disposal. In addition, we applied the inversion method to NaI data with the same $\xi$. In this manner, the structures for both IS species will undergo the same filtering. Nevertheless, since the NaI data are more numerous than HI data, it remains that the cloud surface limits are better defined for NaI data.

More details on the method are given in Appendix A.

\section{The data}

\subsection{Data sources}

We take into account the following databases for $\mathrm{NaI}$ equivalent widths:

- Welsh et al. (1994) data (200 stars);

- Sfeir et al. (1999) data (204 stars).

For the stars not available in these databases, we take the Garcia (1991) data (602 stars). The Garcia (1991) compilation contains old measurements which are very unprecise due to low resolution, telluric contamination or companion star lines, etc. In the case where more recent data have been recorded, we take them preferentially.

For HI column densities, we consider:

- Diplas et al. (1994) data (454 stars).

For the stars not available in this precedent database, we take the Fruscione et al. (1994) data (586 stars).

For NaI, we have 720 IS stars with a heliocentric distance lower than 1500 pc (Fig. 1), and for HI, 567 stars belonging to the $2500 \mathrm{pc}$ radius sphere (Fig. 2). Note that column densities for the further stars contribute to give information of the nearer ISM, as shown by Eq. (1). For this reason, we take into account the distant stars in our analysis.

We use the Hipparcos parallaxes to determine stellar distances and distance errors for stars as far as 200 pc. For stars further than $200 \mathrm{pc}$, we consider the Neckel et al. (1980) photometry distances. We determine distances

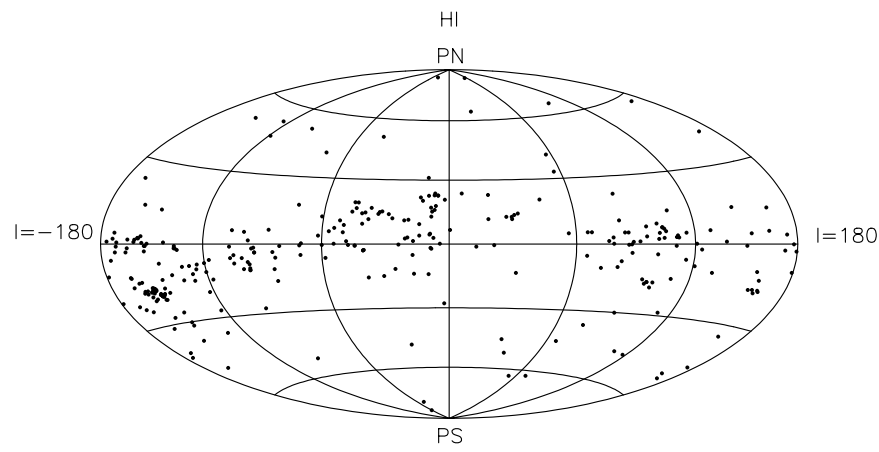

Fig. 2. Projection of the HI target stars on the celestial sphere for the stars closer than $1.5 \mathrm{kpc}$

from the Hipparcos data, if the relative errors $\left(\sigma_{\pi} / \pi\right)$ are smaller than 0.3 , since for photometric distances, the relative error is about 0.3 .

\subsection{Estimation of column densities from the equivalent widths}

Column densities $N$ can be determined from equivalent widths $W_{\lambda}$ by using a theoretical curve of growth. Such a function, however, applies to the ideal case of a unique, homogeneous velocity component. Reality is different: Sfeir et al. (1999) have shown that, when the analysis of high resolution spectra provides both $N$ and $W_{\lambda}$, the resulting set of available data points $W_{\lambda}$ and $N$ is best fitted by the theoretical curve of growth for a unique cloud at $T=10000 \mathrm{~K}$. Because the absorbing media are much cooler $(T<1000 \mathrm{~K})$, this empirical behavior reflects the fact that when saturation begins there is already more than one velocity component.

In our work, we will assume that saturation is only apparent and consider that $N$ is proportional to $W_{\lambda}$ (we will remain on the linear part of the growth curve).

The relation between the column density, $N$, and the equivalent width, $W_{\lambda}$, in the optically thin case (optical depth lower than 1: Spitzer 1968) is:

$N\left(\mathrm{~cm}^{-2}\right)=1.1310^{17} \frac{W_{\lambda}(\mathrm{m} \AA)}{f \lambda^{2}(\AA)}$,

where $\lambda$ is the rest wavelength of the line and $f$ its oscillator strength (as given, for example, by Morton 1991, 1992).

This formulation leads to an underestimation of $N$ for distances larger than $150 \mathrm{pc}$ when saturation occurs.

If column densities were underestimated, the gradient of fluctuations of the local density, according to Eq. (2), would behave in the same way. In this case, we can lose some cloud positions but, on the other hand, we are not creating any artificial features during the inversion. 


\subsection{Estimation of data error}

If observational data are biased or errors have been underestimated, we cannot expect to obtain significant results because the inversion technique will try to understand and to reproduce in a coherent model an image of the initial data.

Data errors arise either from measurements of the equivalent width, from the conversion of $W_{\lambda}$ to column densities, or from distances obtained from Hipparcos database.

The distance error can be considered as an absorption error, $\sigma_{N_{d}}$. Let us first write the absorption as:

$N=\bar{\rho} d$,

where $\bar{\rho}$ is the mean absorption by distance unit and $d$ the distance of the considered star.

Assuming that $\bar{\rho}$ is known, we write:

$\sigma_{N_{d}}=\bar{\rho} \sigma_{d}=N \frac{\sigma_{d}}{d}$

$d=\frac{1}{\pi}$

where $\pi$ is the Hipparcos parallax. This yields:

$\sigma_{N_{d}}=N \frac{\sigma_{\pi}}{\pi}$

This relation gives the column density error expected from the parallax error. If we consider that the relative column density error is about $25 \%$, the total error for $\mathrm{NaI}$ column densities, when distances are determined from Hipparcos parallaxes, can be written as:

$\sigma_{N}=\sqrt{(0.25 N)^{2}+\left(\frac{\sigma_{\pi}}{\pi} N\right)^{2}+\left(6.010^{10}\right)^{2}}$.

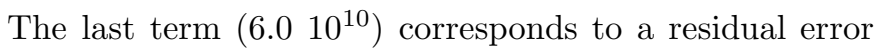
avoiding a null error for low absorption stars.

When photometric distances are taken into account for distances greater than 200 pc, the total error will be:

$\sigma_{N}=\sqrt{(0.25 N)^{2}+(0.3 N)^{2}+\left(6.010^{10}\right)^{2}}$.

Here, the relative photometric distance error is taken equal to $30 \%$ (Neckel et al. 1980) .

As an example of dispersion values, Fig. 3 shows equivalent width $(W)$ data from the Garcia (1991) database. Assuming a linear relation between $W$ and $N$, errors will follow the same behaviour on $W$ and $N$.

For HI, we consider the error on the column densities as given in the Diplas \& Savage (1994) catalogue and the error on distances, the same as for the NaI.

\section{Estimation of the local density}

\subsection{The spatial 3-D density distribution of the ISM}

The inversion of $\mathrm{NaI}$ and $\mathrm{HI}$ column densities allows us to obtain the 3-D density distribution of each of these IS
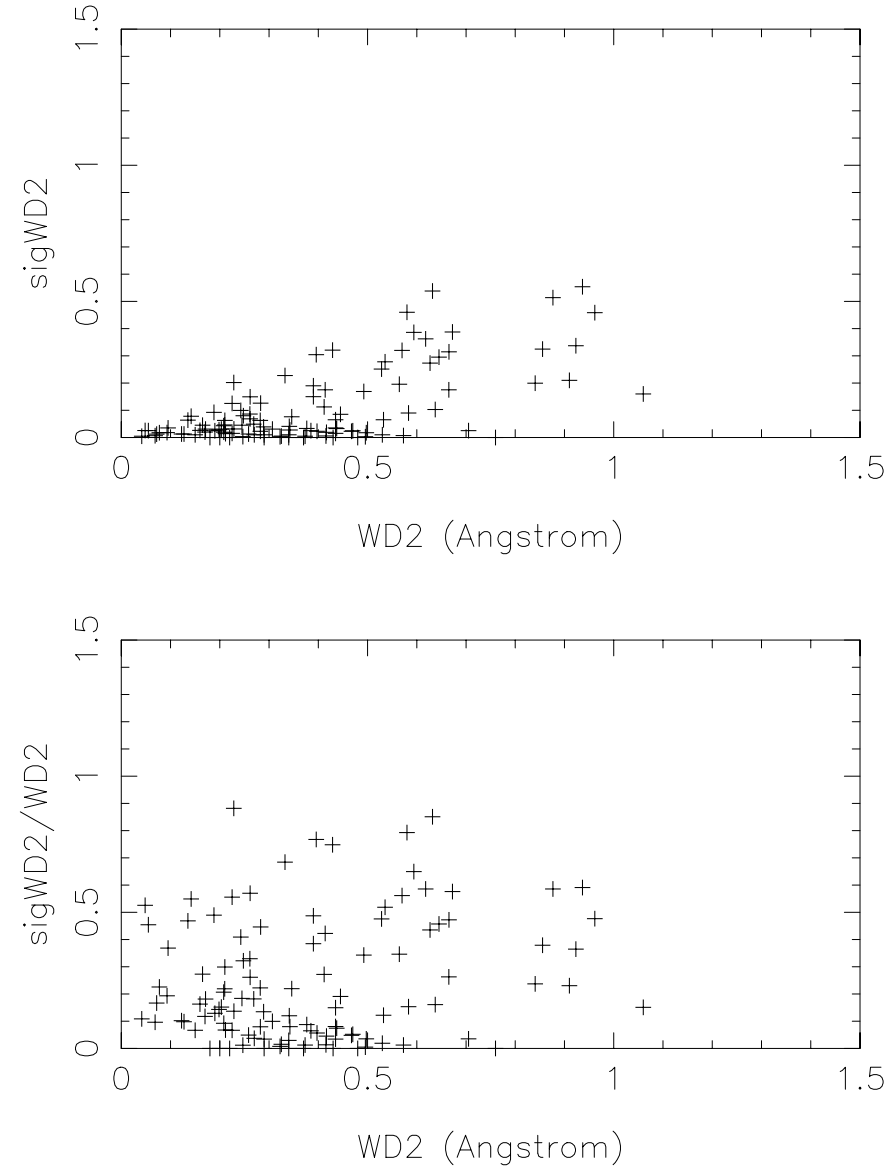

Fig. 3. NaI equivalent width dispersion (top) and relative dispersion (bottom) computed from different authors (Garcia database)

species. For the smoothing lengths $\xi$ equal to 40 and 60 pc, the reduced $\chi^{2}$ obtained are 1.5 and 1.9 respectively, and the local $\mathrm{NaI}$ density varies between $810^{-11}$ and $810^{-8}$. The reduced $\chi^{2}$ obtained for HI is 1.8 for $\xi=60 \mathrm{pc}$ and the local HI density varies from $310^{-2}$ to $7 . \mathrm{cm}^{-3}$.

Notice that the density contrast is higher for the NaI, because of the small smoothing length and the large value of $\sigma_{\alpha}$.

In order to visualise these spatial distributions, we present 6 figures:

- the galactic plane: for NaI with smoothing lengths $\xi$ of $40 \mathrm{pc}$ (Fig. 4) and for $\mathrm{NaI}$ and $\mathrm{HI}$ with $\xi$ of $60 \mathrm{pc}$ (Fig. 6);

- a bundle of planes perpendicular to the galactic plane, centered on the Sun and uniformly separated by $15^{\circ}$ : for $\mathrm{NaI}$, with $\xi$ equal to $40 \mathrm{pc}$ (Fig. 5) and for $\mathrm{HI}$ and $\mathrm{NaI}$, with $\xi$ equal to 60 pc (Fig. 9);

- 3-D density distribution (Figs. 7 and 8).

All the figures are sections of cubes with sides of 500 pc. The darkest regions of the maps correspond to, respectively, NaI density greater than $310^{-9} \mathrm{~cm}^{-3}$ and HI density greater than $1.5 \mathrm{~cm}^{-3}$. 

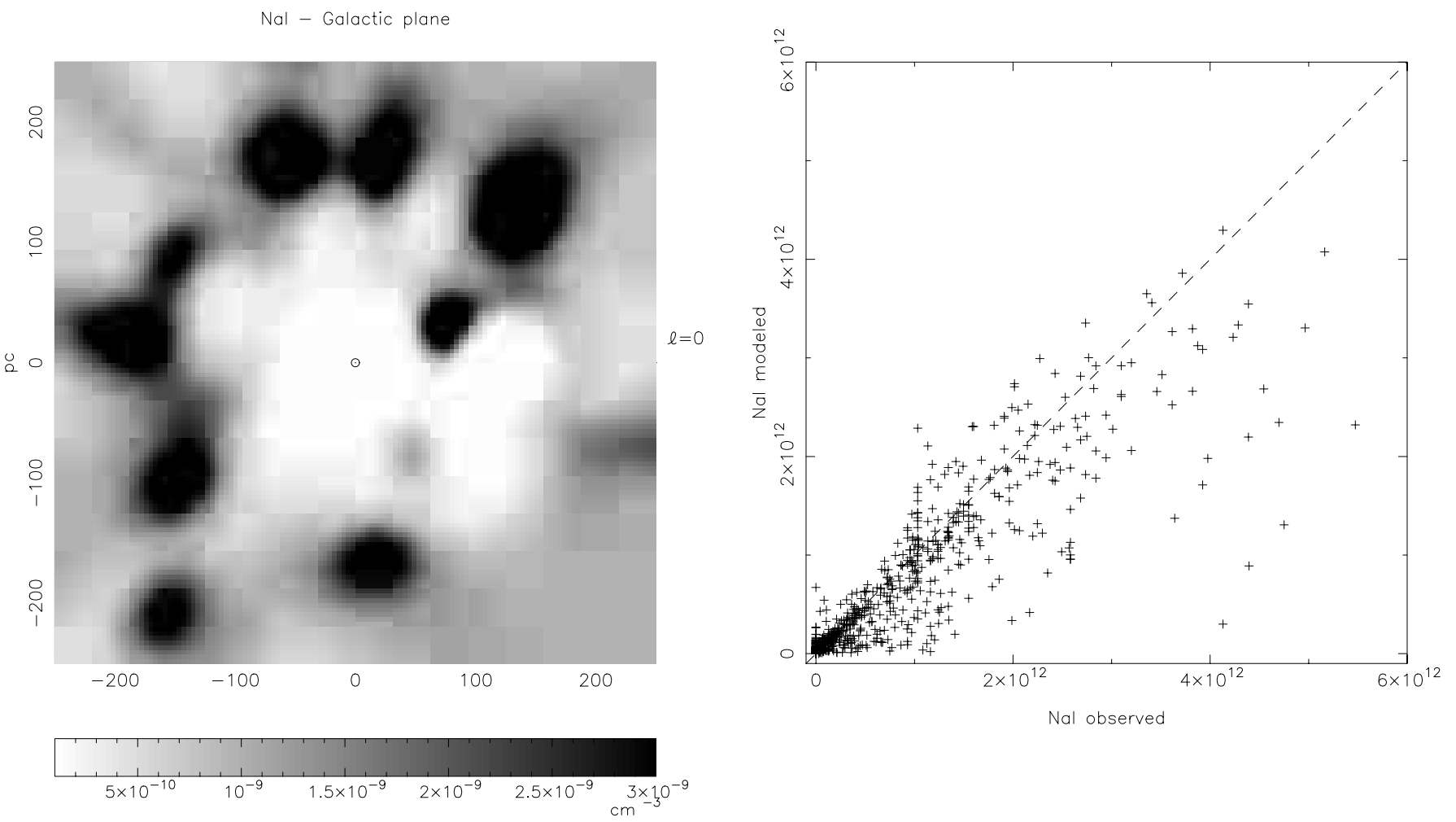

Fig. 4. Cross section of the 3-D IS density distribution by the galactic plane. Left panel: NaI clouds for the smoothing length $\xi=40 \mathrm{pc}$. The Sun is in the center of the map, the Galactic center $(l=0)$ is at the right side of the figure. The right panel shows the quality of the fit

Four general conclusions emerge from the analysis of these sections:

- IS matter is well distributed in compact clouds or in cloud complexes, defining cavities and probably empty tunnels between them;

- there is also a general diffuse matter covering large scale volumes;

- even if we had access to the complete set of all stars in the corresponding space volume, we could not know exactly the IS distribution of matter in detail, because we compute here mean spatial densities over scales of order $\xi$. Local values on scales smaller than $\xi$ are difficult or indeed impossible to estimate for finite data sets.

\subsection{Data adjustment and outliers}

Outliers are spotted by comparison of the column density data with column density values given by the model.

We can split these outliers into three categories:

- erroneous data that the inverse method cannot interpret in terms of the neighbouring values; the inverse method will suggest other values in agreement with those of the surrounding (category 1);

- correct data but with a very anomalous value in relation to those of the neighborhood (category 2); the inverse method will proceed as above;
- Finally, there are correct data but taken as anomalous by the inverse method due to their location (category 3 ).

Tables 1 and 2 give the list of the stars that belong to the categories 1 and 2 according to the criteria $0.5<$ $N_{\text {obs }} / N_{\text {model }}<2.0$ together with the median column density and standard deviation of their neighbourhood.

The majority of the stars whose reconstructed column density does not agree with observed data belong to the third category. We see that those stars are located next to cloud or hole edges and, because of the smoothing length, will induce errors in the reconstruction of the column density. This can be easily understood if we look at such a star in the $1 \mathrm{D}$ case. With the smoothing that is applied to the maps, the matter located next to the edge of a cloud (or hole) will be distributed over a distance corresponding to the smoothing length $\xi$. The density, gradient will be lowered at the edge, in relation to the real gradient, and then will affect the column density, since IS matter will be added or removed from the path.

Nevertheless, not all stars belong to that category and there remain stars where the reconstructed column density does not fit the observed one, even if those stars are not located near a cloud edge. In this case, the fact that the inverse method cannot reproduce the observed data is mostly due to the surrounding of the target star. If there are other targets in a sphere of radius equal to the smoothing length, the inverse technique will compare all the data 

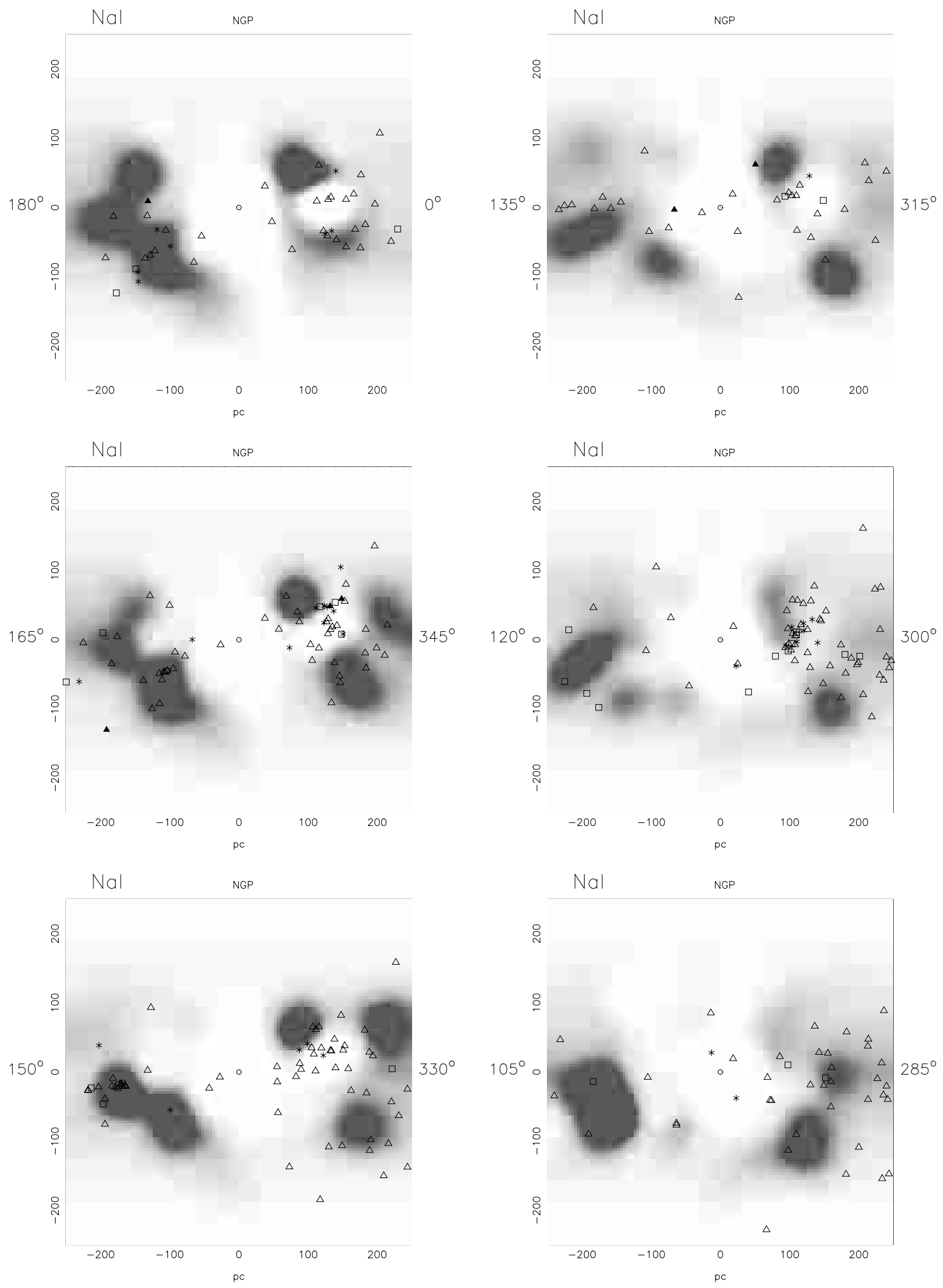

Fig. 5. Different plan cross sections of NaI clouds perpendicular to the galactic plane. The Sun is in the center of each map. The smoothing length $\xi=40$ pc. Symbols are: open triangle, B7V-B9V stars; square, B4V-B6V stars; star, B2V-B3V stars; black triangle, $\mathrm{O} 2 \mathrm{~V}-\mathrm{B} 1 \mathrm{~V}$ stars. The Strömgren sphere around each spectral type depends on the temperature of the stars

and try to reproduce the majority of them. If some of the data is not in the same range as its surrounding (either because of small clouds in front of the target which will lead to an increase of $N$ for that target compared to the neighbouring ones, or because of a wrong measurement) the method will treat it as an outlier. 



Fig. 5. continued

\section{Results and Discussion}

\subsection{Comparison of the Nal 3-D distribution with the HI 3-D one}

We have analysed IS NaI and HI, which is identified only through particular IS lines characterised by differ-

ent ranges of temperature. For temperatures outside these ranges, other radiation and matter interactions could inform us about the presence and extent of these species in the ISM.

The first ionisation level of $\mathrm{NaI}$ is below that of $\mathrm{HI}$. It is expected that the $\mathrm{NaI}$ will be present in very low 
HI - Galactic plane
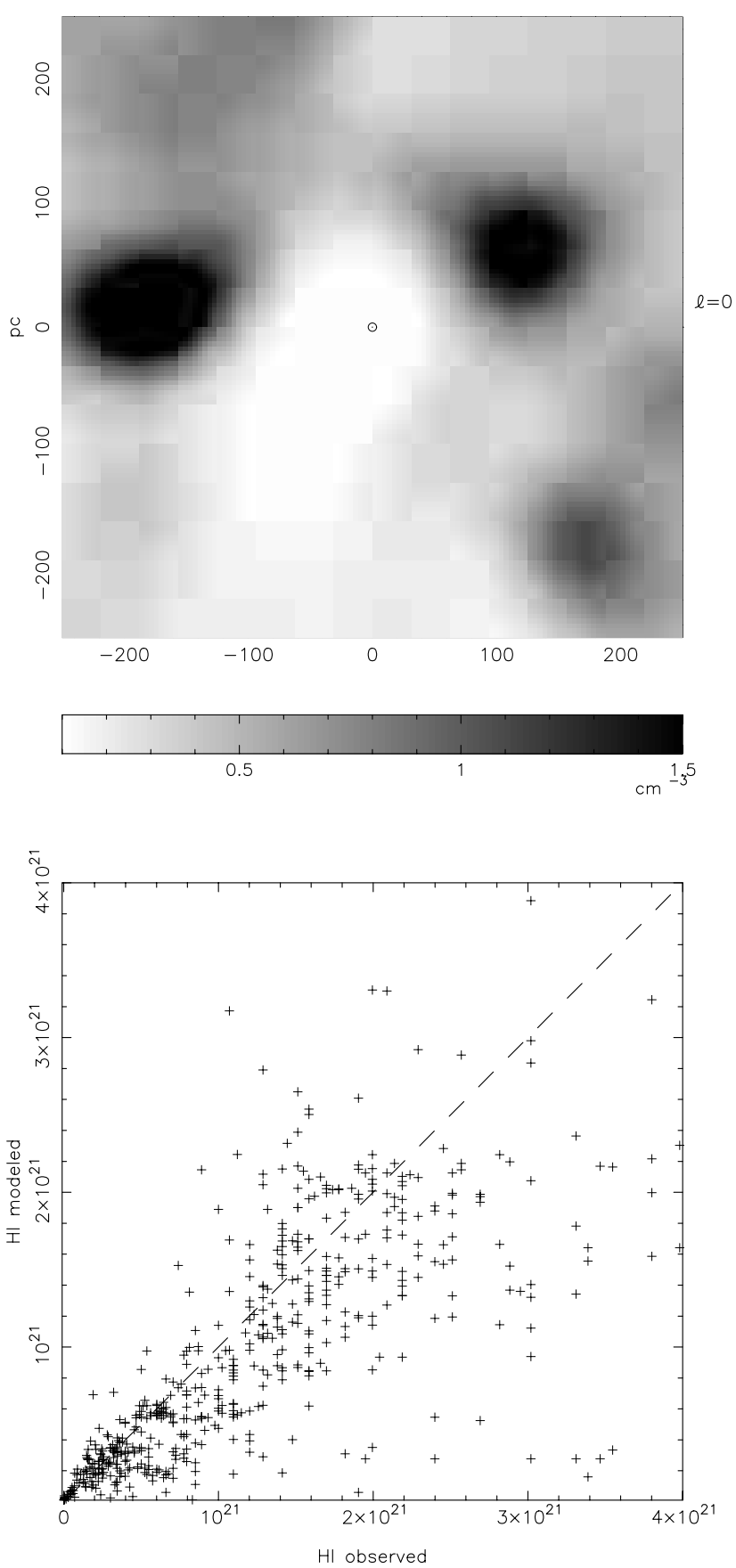

$\mathrm{Nal}$ - Galactic plane
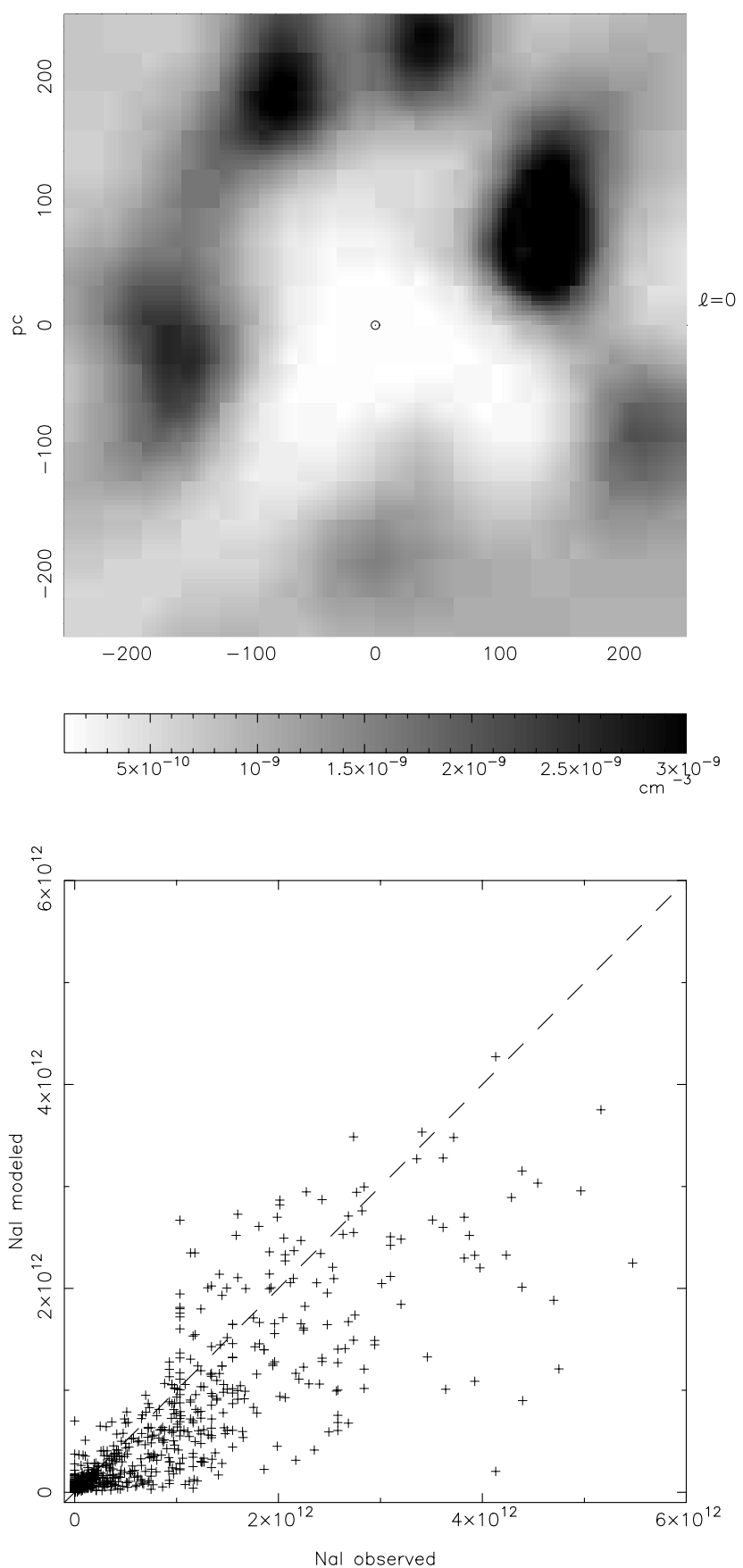

Fig. 6. Comparison between cross sections of HI and NaI clouds by the galactic plane (top). The smoothing length is $\xi=60$ pc. The Sun is in the center of each cross section. Bottom panels: comparison of HI and NaI column densities derived by our model with those derived from observations

temperature clouds. As soon as the temperature rises, NaI becomes ionized, even thought the HI remains neutral. Thus HI may have a larger spatial extension compared to NaI. This is observed in Fig. 9: for example, the empty cavity around the Sun, the so-called Local Bubble (hereafter LB), appears smaller in NaI than in HI.

As viewed in NaI with $\xi$ equal to 40 pc (Fig. 5), the LB appears more or less spherical, as do the other observed cavities: nevertheless, some meridian sections show the LB elongated perpendicular to the galactic plane.

Through the different sections in Fig. 9, and having in mind that $\xi=60 \mathrm{pc}$, we can see two annular structures centered more or less on the empty cavity around the Sun and tilted in relation to the galactic plane, with a radius varying from 100 to $200 \mathrm{pc}$. The slope also varies from $20^{\circ}$ over the section $l=0-180^{\circ}$ to $45^{\circ}$ for section $l=90-270^{\circ}$ and $60^{\circ}$ for section $l=60-240^{\circ}$ to section $l=15-195^{\circ}$. 
Table 1. Stars with an observed column density that does not agree with the neighbouring values (category 1)

\begin{tabular}{|c|c|c|c|c|c|c|c|c|c|c|}
\hline $\mathrm{HD}$ & $l\left(^{\circ}\right)$ & $b\left(^{\circ}\right)$ & $\mathrm{Sp}$ & $\pi$ (mas) & $\begin{array}{l}N_{\text {model }} \\
\left(10^{10}\right)\end{array}$ & $\begin{array}{l}N_{\text {obs }} \\
\left(10^{11}\right)\end{array}$ & $N_{\text {obs }} / N_{\text {model }}$ & $\begin{array}{l}\text { number of stars } \\
\text { at less than } 1 \xi\end{array}$ & $\begin{array}{l}N_{\text {med }}^{(a)} \\
\left(10^{10}\right)\end{array}$ & $\begin{array}{l}\sigma_{N_{\text {med }}}^{(b)} \\
\left(10^{10}\right)\end{array}$ \\
\hline 2772 & 120.05 & -8.24 & $\mathrm{~B} 8 \mathrm{Vn}$ & 9.20 & 7.35 & 4.04 & 5.50 & 5 & 4.96 & 9.79 \\
\hline 10250 & 127.21 & 8.18 & B9V & 11.62 & 4.64 & 1.01 & 2.18 & 5 & 0.00 & 11.5 \\
\hline 21278 & 147.52 & -6.19 & B5V & 5.72 & 112. & 23.5 & 2.10 & 3 & 72.3 & 91.7 \\
\hline 23509 & 281.35 & -42.84 & $\mathrm{~A} 5 \mathrm{Vm} \ldots$ & 4.68 & 107. & 25.8 & 2.41 & 2 & 76.5 & 13.9 \\
\hline 61497 & 158.51 & 29.52 & A3IVn & 13.75 & 2.56 & 0.70 & 2.74 & 3 & 0.00 & 4.96 \\
\hline 109401 & 288.17 & 73.30 & A5 & 9.43 & 3.89 & 1.29 & 3.32 & 3 & 0.00 & 0.00 \\
\hline 124367 & 314.13 & 3.96 & B4Vne & 6.71 & 10.9 & 9.30 & 8.53 & 1 & 0.00 & - \\
\hline 129685 & 327.74 & 22.15 & $\mathrm{~A} 0 \mathrm{~V}$ & 15.76 & 1.39 & 2.14 & 15.40 & 3 & 0.00 & 3.31 \\
\hline 136504 & 329.23 & 10.32 & B2IV-V & 6.47 & 6.87 & 10.8 & 15.72 & 5 & 0.00 & 3.46 \\
\hline 141569 & 4.18 & 36.92 & B9 & 10.10 & 45.7 & 9.71 & 2.13 & 8 & 9.34 & 73.9 \\
\hline 141637 & 346.10 & 21.71 & $\mathrm{~B} 1.5 \mathrm{Vn}$ & 6.25 & 44.2 & 11.3 & 2.56 & 4 & 64.8 & 65.9 \\
\hline 159532 & 347.14 & -5.98 & F1II & 11.99 & 15.3 & 3.55 & 2.32 & 4 & 0.00 & 0.00 \\
\hline 159561 & 35.89 & 22.57 & A5III & 69.84 & 0.01 & 0.26 & 180.42 & 14 & 0.00 & 0.00 \\
\hline 167666 & 3.69 & -5.92 & $\mathrm{~A} 5 \mathrm{~V}$ & 8.21 & 22.4 & 12.0 & 5.36 & 6 & 16.1 & 96.2 \\
\hline 188350 & 40.55 & -13.92 & A0III & 9.45 & 20.6 & 11.3 & 5.49 & 5 & 4.39 & 36.0 \\
\hline 23274 & 116.97 & 5.67 & $\mathrm{~A} 1 \mathrm{Vn}$ & 10.96 & 5.20 & 1.15 & 2.21 & 5 & 0.00 & 11.3 \\
\hline
\end{tabular}

(a) Median observed column density of the neighbouring stars.

(b) Standard deviation.

Table 2. Stars with an observed column density that cannot be reproduced (category 2)

\begin{tabular}{|c|c|c|c|c|c|c|c|c|c|c|}
\hline $\mathrm{HD}$ & $l\left(^{\circ}\right)$ & $b\left(^{\circ}\right)$ & $\mathrm{Sp}$ & $\pi$ (mas) & $\begin{array}{l}N_{\text {model }} \\
\left(10^{11}\right)\end{array}$ & $\begin{array}{l}N_{\text {obs }} \\
\left(10^{12}\right)\end{array}$ & $N_{\text {obs }} / N_{\text {model }}$ & $\begin{array}{l}\text { number of stars } \\
\text { at less than } 1 \xi\end{array}$ & $\begin{array}{l}N_{\text {med }}^{(a)} \\
\left(10^{11}\right)\end{array}$ & $\begin{array}{l}\sigma_{N_{\text {med }}}^{(b)} \\
\left(10^{12}\right)\end{array}$ \\
\hline 26594 & 287.20 & -37.31 & $\mathrm{~A} 4 \mathrm{~V}$ & 4.36 & 4.62 & 1.16 & 2.51 & 1 & 6.66 & - \\
\hline 35575 & 204.16 & -19.71 & B3V & 4.12 & 4.89 & 1.24 & 2.54 & 1 & 8.51 & - \\
\hline 101075 & 135.50 & 51.13 & A0 & 4.22 & 2.41 & 1.17 & 4.86 & 0 & - & - \\
\hline 151749 & 60.61 & 39.85 & B9 & 6.48 & 1.63 & 0.38 & 2.34 & 1 & 0.80 & - \\
\hline 154445 & 19.30 & 22.93 & $\mathrm{~B} 1 \mathrm{~V}$ & 4.26 & 3.14 & 1.11 & 3.54 & 0 & - & - \\
\hline 155379 & 358.98 & 8.23 & $\mathrm{~A} 0 \mathrm{~V}$ & 8.81 & 0.84 & 0.43 & 5.14 & 8 & 2.25 & 1.25 \\
\hline 203532 & 309.46 & -31.74 & B3IV & 4.01 & 4.28 & 1.46 & 3.41 & 0 & - & - \\
\hline 205637 & 31.94 & -44.99 & B3V:p & 4.92 & 2.36 & 4.13 & 17.50 & 0 & - & - \\
\hline 208886 & 15.64 & -52.59 & B5III & 3.54 & 2.29 & 1.41 & 6.16 & 0 & - & - \\
\hline
\end{tabular}

(a) Median observed column density of the neighbouring stars.

(b) Standard deviation.

The other annular structure appears incomplete and is visible only from section $l=45-225^{\circ}$ to section $l=15^{-}$ $195^{\circ}$. It is tilted over $15^{\circ}$ in the direction opposite to the first one.

For NaI, similar annular structures than those for HI are seen, but the comparison of Fig. 5 ( $\xi=40$ pc) with Fig. $9(\xi=60 \mathrm{pc})$ shows that the annular structures split into many clouds when we allow for lower correlation lengths. The spatial 3-D distribution of IS NaI and
HI shows some superposition of clouds, but it also shows differences in many regions (indicating either that the concentration of different species are different or that differences in temperature mask the presence in rather constant ratios of all components of the ISM). More generally, we could also suggest that these annular structures are concentrated IS matter over a sort of coronal volume with an ellipsoidal shape centered more or less around the Sun. The origin and meaning of this IS "superstructure" can 


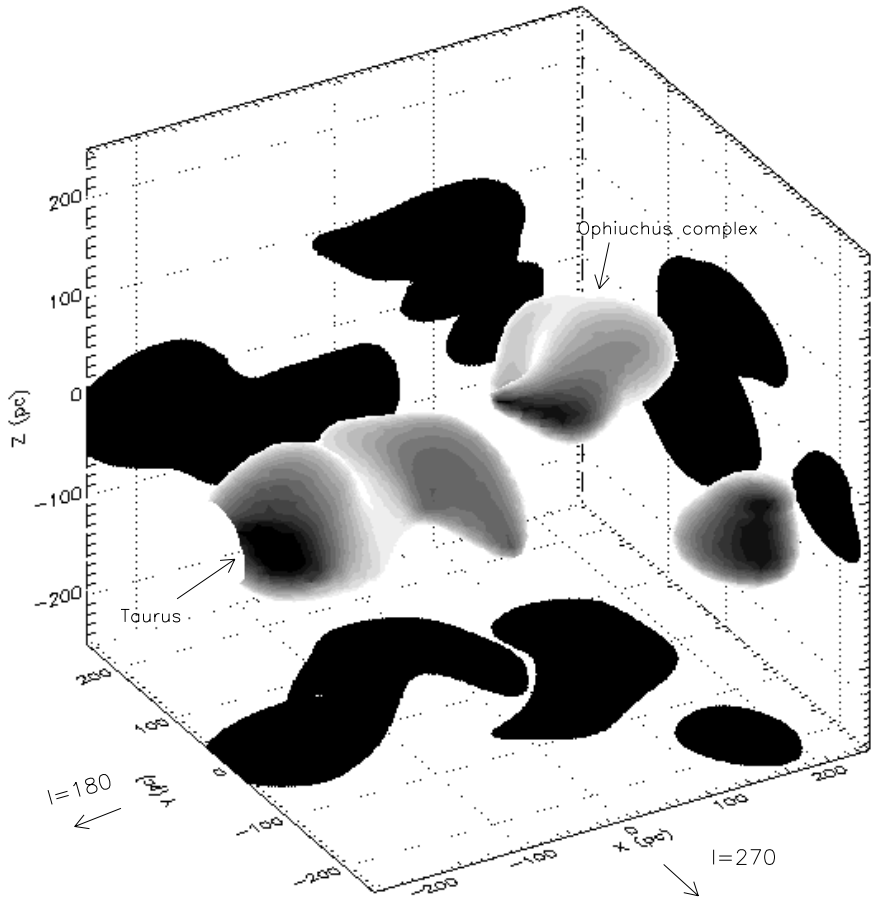

Fig. 7. 3-D mapping of HI clouds around the Sun for contour level $1 \mathrm{~cm}^{-3}$. The structures are projected as black spots over the three fundamental planes

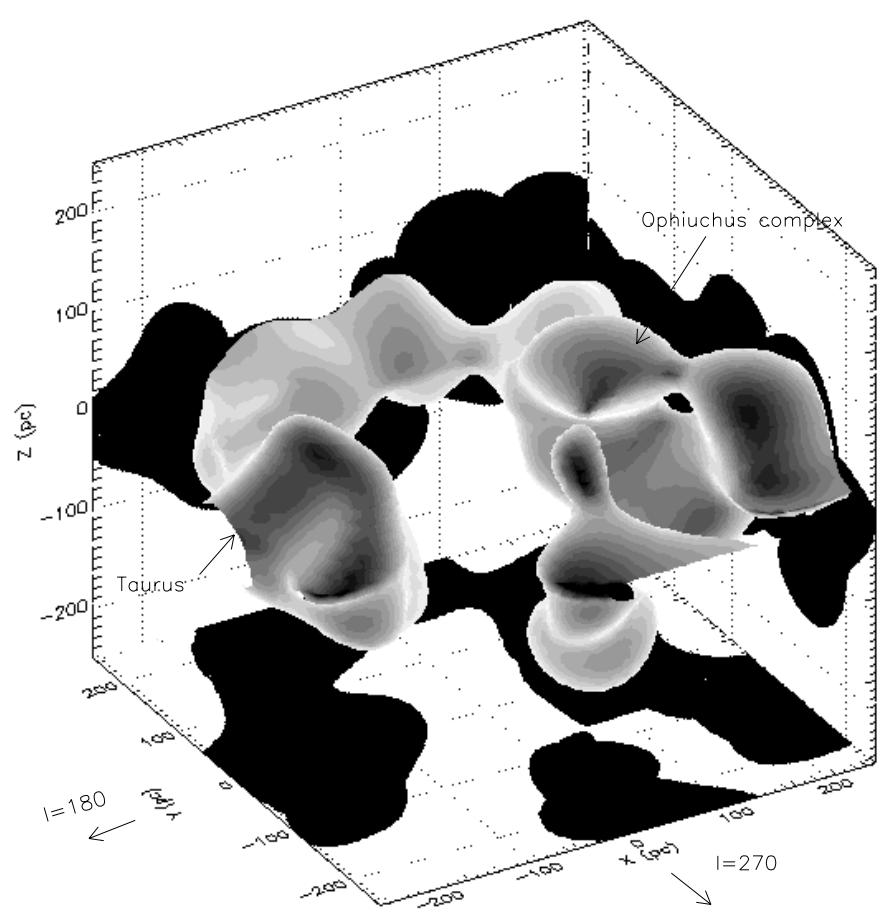

Fig. 8. 3-D mapping of NaI clouds around the Sun for contour level $110^{-9} \mathrm{~cm}^{-3}$. The structures are projected as black spots over the three fundamental planes

only be the object of scenario attempts, such as the resulting effects of supernova explosions creating the LB, the Loop I cavity and the Gould Belt.
An important result emerging from our spatial 3-D density distribution for the NaI data using $\xi=40 \mathrm{pc}$ is that other cavities appear more or less well defined:

- one associated with the Ophiuchus Complex $\left(l<30^{\circ}\right.$ and $\left.l>330^{\circ}\right)$, the Loop I and Sco-Cen Association $\left(300^{\circ}<l<330^{\circ}\right)$ and the loop IV $\left(280^{\circ}<l<340^{\circ}\right)$, in section $l=15^{-195^{\circ}}$, and from sections $l=0-180^{\circ}$ to $l=105-285^{\circ}$, with a size a half or less that of the $\mathrm{LB}$;

- one associated with Per-Tau $\left(140^{\circ}<l<180^{\circ}\right)$, from sections $l=0-180^{\circ}$ to $l=150-330^{\circ}$.

In particular, the LB appears asymmetric both in HI and $\mathrm{NaI}$ sections and more extended in $\mathrm{HI}$ than in $\mathrm{NaI}$.

Another important result concerning the analysis of $\mathrm{NaI}$ data is that the LB is practically connected with the medium outside the galactic plane through "corridors" or "chimneys" scarcely tilted from the perpendicular to the galactic plane, as seen in sections $l=0-180^{\circ}$ and $l=$ $165-345^{\circ}$ (to the bottom), from sections $l=150-330^{\circ}$ to $l=60-240^{\circ}$ (to the top) and from sections $l=45-225^{\circ}$ to $l=15-195^{\circ}$ (to the bottom). These "chimneys" are really regions with poor matter density and they are not surrounded by cloud complexes. There are other regions separating clouds with minor densities, but they do not seem to have the same character as those "corridors".

Using ROSAT data, Egger \& Aschenbach (1996) discovered a ring-like structure deficient in the X-ray around Sco-Cen. The intersection of a like-ring absorption structure with different vertical planes will give a two nearby circle-like clouds, which is what we see in the IS NaI maps (Figs. 5 and 9) in the directions $0^{\circ}<l<315^{\circ}$ but it is not well-defined for HI (Figs. 9). This is surely due to the large correlation length used in the model.

The Gould Belt plane is tilted over $20^{\circ}$ (up to $l=0^{\circ}$ and down to $l=180^{\circ}$ ) with respect to the galactic plane, following quasi exactly the angle represented by the line joining the two important cloud sections in both $\mathrm{HI}$ and NaI (Fig. 9). In Fig. 5 the inclination globally responds to the line joining the barycenter of the two opposite group of clouds shown in section $l=0-180^{\circ}$.

\subsection{Comparison with previous results}

As previously found by many authors (Welsh et al. 1994; Sfeir et al. 1999 and references therein), there is consistent evidence for a neutral, gas-free cavity (the Local Bubble) around the Sun, a result that we find again using a large database of $\mathrm{NaI}$ and HI IS absorptions.

Welsh (1991) and Welsh et al. (1994) conclude that there is an IS tunnel of low NaI density $\left(\rho_{\mathrm{HI}}<0.05 \mathrm{~cm}^{-3}\right)$ $50 \mathrm{pc}$ wide and $240 \mathrm{pc}$ long in the direction of $\beta \mathrm{CMa}$ $\left(l=235^{\circ}, b=-20^{\circ}\right)$, but from our 3 -D projections, we find that more conspicuous "tunnels" probably exist in many other directions. Of course, our results depend on the $\xi$ value and in one specific direction, a void tunnel with size less than the $\xi$ value could be smeared onto its 



Fig. 9. Comparison between cross sections of HI and NaI clouds The Sun is in the center of each section. The smoothing length is $60 \mathrm{pc}$

surrounding ISM, giving as a solution a larger volume with a lower spatial density.

In Fig. 10 we show that the extinction (in magnitude units) in the direction of $\beta \mathrm{CMa}$ is relatively constant for a distance of the order of $250 \mathrm{pc}$, with little scatter. Then, the extinction grows slowly to a mean value of 0.03 . We take into account all the stars of our database (54 stars) at the interior of a solid angle of $5^{\circ}$ around the $\beta \mathrm{CMa}$ direction up to $400 \mathrm{pc}$. That result confirms a relatively void space in this direction. 

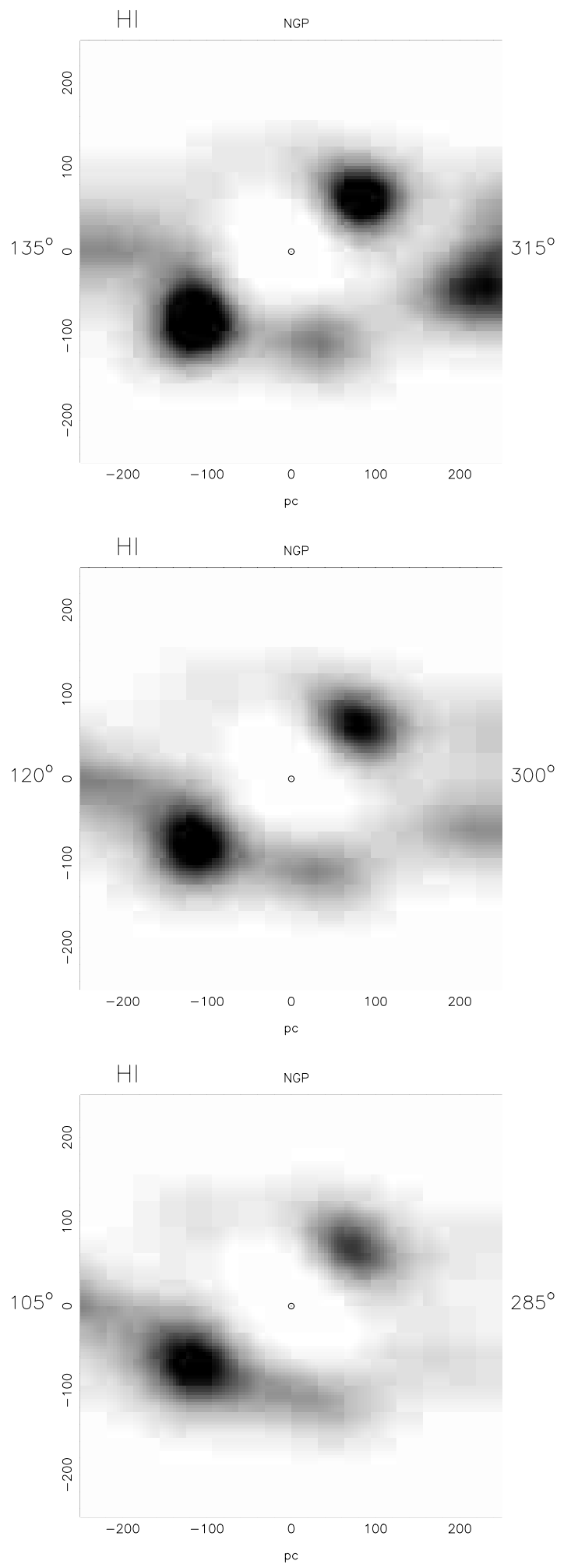

Fig. 9. continued

The tunnel deduced by Welsh et al. (1994) at $l=330^{\circ}$, $b=12^{\circ}$ (towards Lupus-Norma) appears, in our plots, as a region of lower density connecting with a cavity corresponding to the Loop I bubble. The extinction plot in this direction (also Fig. 10) shows a low extinction level $(E(b-y) \simeq 0.02)$. The problem is to know if these tunnels
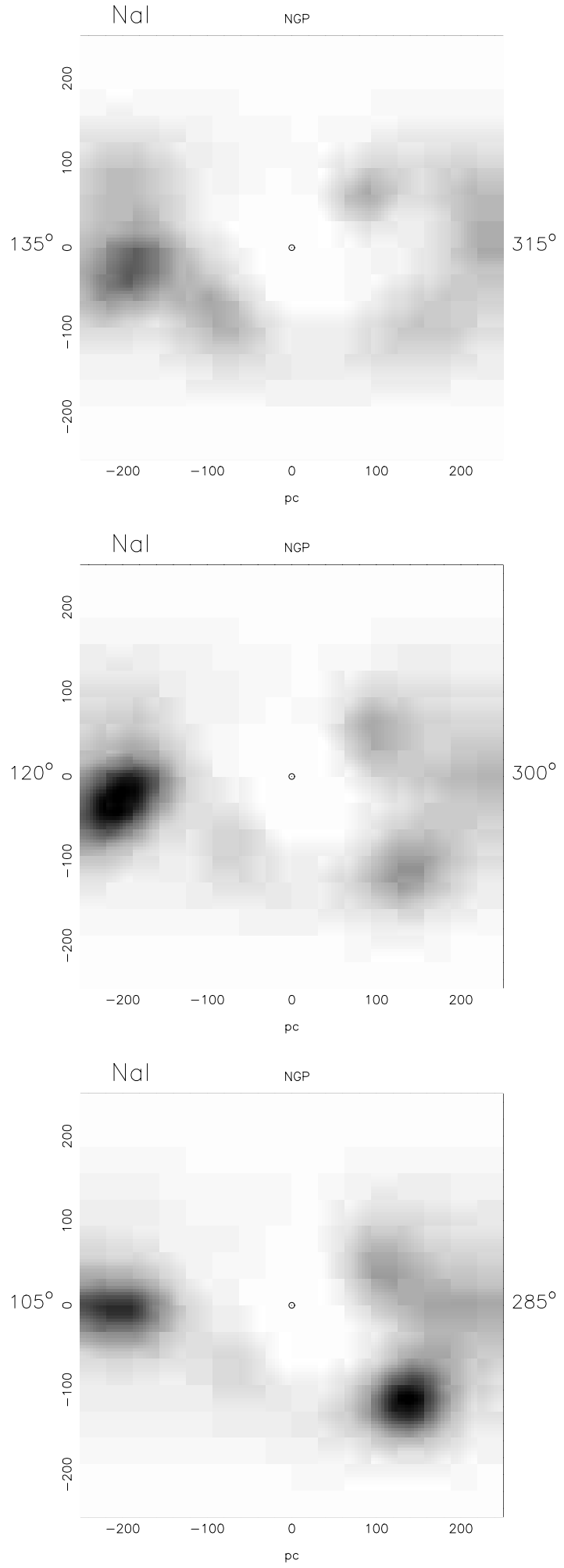

are real "structures" or if they are either simply relatively empty inter-cloud space, or, in our case, a result of the correlation length which we used.

From the comparison of the spatial 3-D density distributions of $\mathrm{NaI}$ and $\mathrm{HI}$, we can place constraints on the $\rho_{\mathrm{NaI}} / \rho_{\mathrm{HI}}$ density ratio. However, the bias obtained on the 



Fig. 9. continued

determination of the $N_{\mathrm{NaI}}$ density columns from the equivalent widths provides a lower limit of this ratio.

The densities (Fig. 13) for both neutral species suggest a factor of proportionality larger than $1.110^{-9}$, but the discrepancies between cloud distribution in each species (Fig. 9) prevent the estimation of a general factor ratio valid for any spatial direction, at least for distances less than 300 pc around the Sun. The correlation seen Fig. 13 is given for a smoothing length $\xi=60$ and cannot be applied to other scales of smoothing. Figure 14 shows the column density correlations given for 60 stars present in both databases. For the weak density values, the correlation 

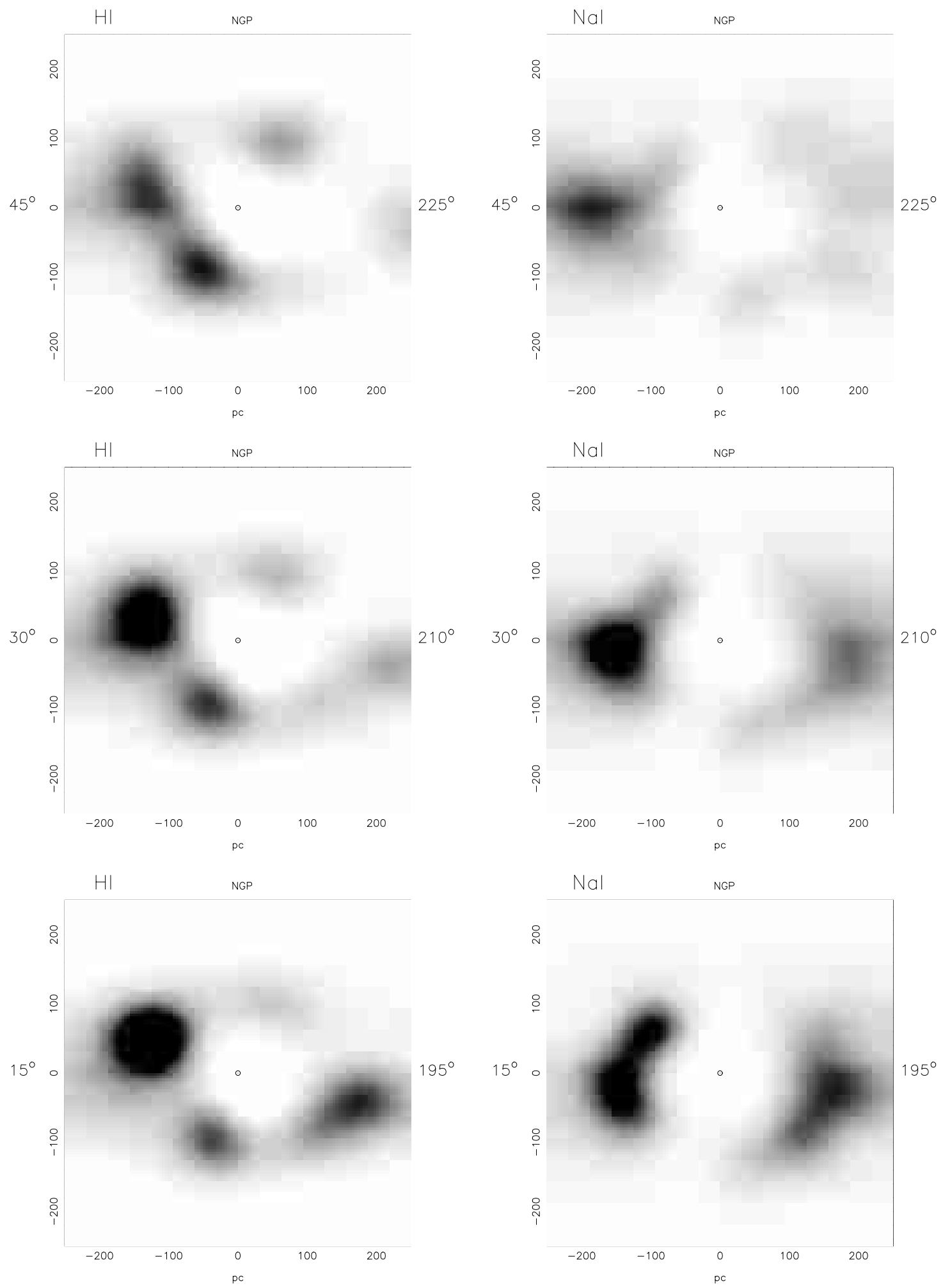

Fig. 9. continued

appears rather scattered. This could be due to the limited number of HI line of sight. Note that the $\rho_{\mathrm{NaI}} / \rho_{\mathrm{HI}}$ density ratio is similar to the column density ratio $N_{\mathrm{NaI}} / N_{\mathrm{HI}}$ found by Ferlet et al. (1985).

Many authors use some estimated population ratios to justify evolutionary or physical scenarios of some proper-

ties of the ISM. Our work shows that, on the contrary, using a more extended database than those used previously, it is not justified to use constant population ratios for IS species, at least for distances lower than $300 \mathrm{pc}$. This is an observational result without any ad hoc hypothesis about the necessity for a single population or column 

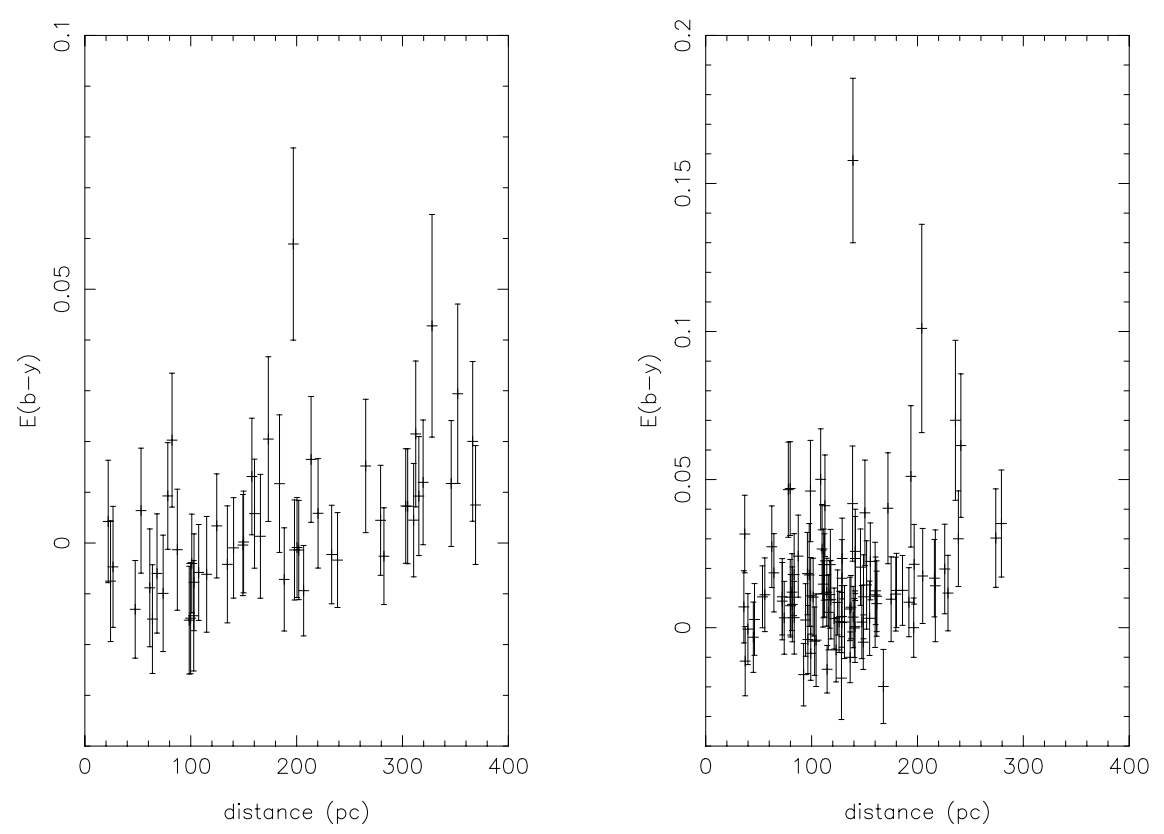

Fig. 10. Extinction $E(b-y)$ versus the distance in the direction of $\beta \mathrm{CMa},-20^{\circ}<b<-5^{\circ}, 225^{\circ}<l<245^{\circ}$ (left panel) and the Loop I, $10^{\circ}<b<20^{\circ}, 320^{\circ}<l<340^{\circ}$ (right panel). The extinction are not significant below 200 pc, which shows clearly the lack of dust in these directions. These directions correspond to "tunnels" with low HI and NaI densities

\section{modeled density column of $\mathrm{Nal}$ at $300 \mathrm{pc}$}

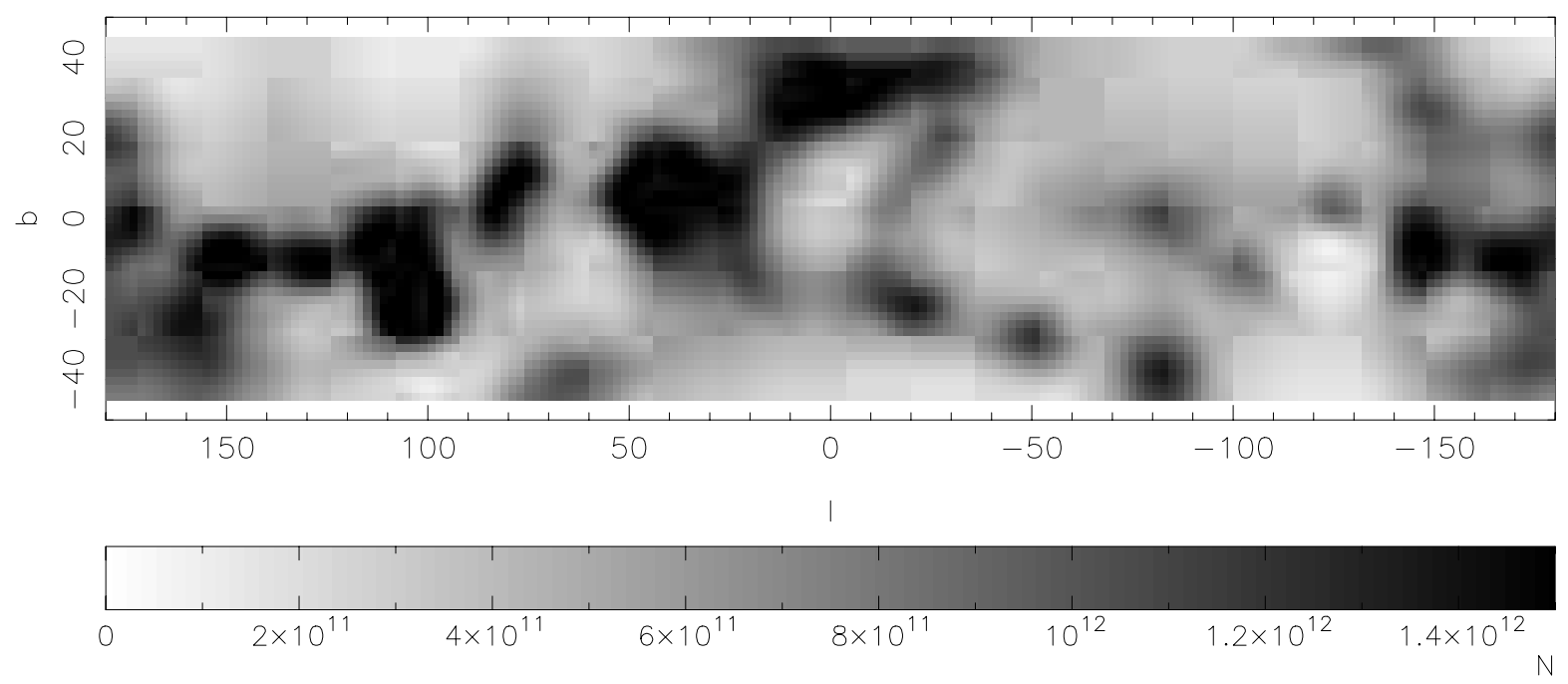

Fig. 11. NaI column densities $\left(\mathrm{cm}^{-2}\right)$ at $300 \mathrm{pc}$ in galactic coordinates. The white areas in the projected sky show the tunnels without $\mathrm{NaI}\left(l=-125^{\circ}\right.$ and $\left.l=-30^{\circ}\right)$

density ratio anywhere. Real ratio variations could be worse, because our results on spatial density values are smoothed through the correlation length parameter.

Individual observations of stars lead to the discovery of inhomogeneities in the ISM. In the direction of $\beta$ CMa and $\epsilon$ CMA the HI column densities are very low: $N_{\mathrm{HI}}<0.01 \mathrm{~cm}^{-2}$ (Gry et al. 1985; Gry et al. 1995). In our work, these stars, as well as $\delta \mathrm{Cyg}$, are placed at the limit of the Local Bubble as seen in HI (see Fig. 6). Because the image of the Local Bubble is different in NaI, these stars appear to have different $\mathrm{NaI}$ densities around them.

The majority of neutral gas in both directions ( $\beta \mathrm{CMa}$ and $\epsilon \mathrm{CMa}$ ) is contained within cloudlets (Dupin \& Gry 1998; Gry et al. 1995), that represent the diffuse local IS cloud (Lallement \& Bertin 1992). 



Fig. 12. Density levels of $\mathrm{HI}$ and $\mathrm{NaI}$ in the Ophiuchus and Taurus regions. In these relatively high-density regions, the correlation between the $\mathrm{HI}$ and the $\mathrm{NaI}$ density is rather good



Fig. 13. Correlation between the $\mathrm{NaI}$ and $\mathrm{HI}$ densities (in atoms $\mathrm{cm}^{-3}$ ). Each loop corresponds to a cloud. The biggest one is the Ophiuchus cloud. The long axis of each loop gives the straight line of correlation between $\rho_{\mathrm{NaI}}$ and $\rho_{\mathrm{HI}}$. Note the dispersion of the slopes

\subsubsection{Photoionisation process in the ISM}

Hot stars can, depending on their temperature, ionize the surrounding IS medium (e.g. Osterbrock 1989). In Fig. 5, stars with a spectral type earlier than B9V are plotted, projected from $10 \mathrm{pc}$ on both side of each cut. These stars have a range of temperature from 10000 to $40000 \mathrm{~K}$. The Strömgren sphere extent depends on the neutral hydrogen density of the medium. For a density of $1 \mathrm{~cm}^{-3}$, the diameter of the Strömgren spheres vary between $25 \mathrm{pc}\left(T_{\text {eff }}=\right.$ $26000 \mathrm{~K})$ and $150 \mathrm{pc}\left(T_{\text {eff }}=40000 \mathrm{~K}\right)$. Indeed, a cluster of young stars with a temperature less than $26000 \mathrm{~K}$ can photoionise the medium in a sphere larger than $25 \mathrm{pc}$ if the medium has a low density, the diameter of the sphere depending on the inverse of the hydrogen density. Note that many relatively cool stars (less than $15000 \mathrm{~K}$ ) are present in compact molecular clouds and do not show photoionisation influences greater than the $40 \mathrm{pc}$ smoothing length. The hottest stars are preferentially located in low NaI density regions or at the boundary of clouds.

The Sco-Centaurus region shows an extended young star association at $300^{\circ}<l<360^{\circ}$ and $-10^{\circ}<b<+30^{\circ}$ These stars are located at heliocentric distances of about 120-160 pc (de Zeeuw et al. 1997). In Fig. 5 at $l=345^{\circ}$ and $d=150 \mathrm{pc}$, the medium is probably strongly ionized by this star association. There seems to be a kind of 


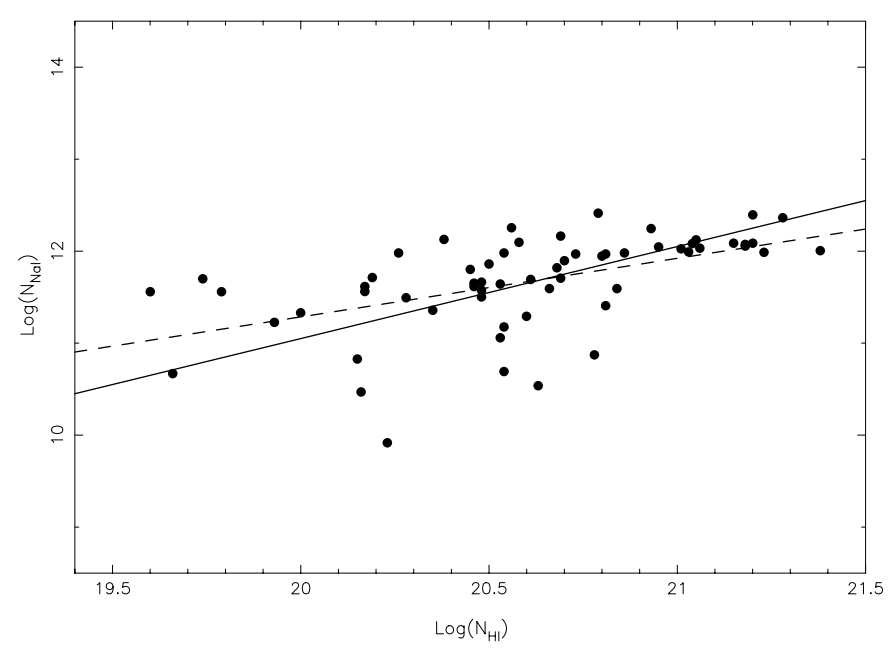

Fig. 14. Correlation between the NaI and HI density column. The dashed line shows the correlation between the data and the full line the correlation given by the density correlation

tunnel surrounding this extended association which joins the loop I.

\subsubsection{Correlation with well-known molecular clouds}

The NaI and HI structures we found can be associated, in some cases, with very well-known molecular or dust clouds (Figs. 7 and 8).

- The largest clouds in the vicinity of the Sun are the Ophiuchus and Taurus complexes which are, respectively, in the direction of the galactic center, above the plane $\left(10^{\circ}<l<-10^{\circ} ; 0^{\circ}<b<+30^{\circ}\right)$ and in the anti-center direction below the plane $\left(335^{\circ}<l<345^{\circ}\right.$; $-20^{\circ}<b<-10^{\circ}$ ). The same structures have been found in NaI and $\mathrm{HI}$ over-density (Fig. 12 at $l=0^{\circ}$ and $l=180^{\circ}$ ). the distance of the more dense regions to the Sun are $170 \mathrm{pc}(\mathrm{HI})$ and $150 \mathrm{pc}(\mathrm{NaI})$ for the Taurus complex and $135 \mathrm{pc}(\mathrm{HI})$ and $130 \mathrm{pc}(\mathrm{NaI})$ for the Ophiuchus complex. These values agree with previous distance estimations from Hipparcos pre-main sequence stars (Bertout et al. 1999);

- Some small high-density molecular clouds are not found in the $\mathrm{NaI}$ and $\mathrm{HI}$ maps. For instance, the Chamaeleon complex $\left(l=300^{\circ} ; b=-20^{\circ}\right)$ is not present in Fig. 5 at $l=300^{\circ}$, probably due to the large smoothing length used;

- Large clouds with a low density are recognized in the area of Vulpecula Sagitta in the NaI maps (Fig. 5, $\left.l=50-60^{\circ}\right)$. These clouds are at a distance of $100-$ 200 pc.

In the HI maps (Fig. 9), these structures are present but very smoothed.

The Eta Carinae complex has a very low density in $\mathrm{NaI}$ (Fig. 5) at $l=285-300^{\circ}$ in a range of distance $100-200 \mathrm{pc}$ on both sides of the galactic plane.

\section{Conclusions}

We apply a powerful and robust inversion technique, similar to that used in tomography analysis, to characterize the 3-D density distribution of two neutral species, NaI and HI. Previous analysis in this domain revealed local IS properties and guided the choice of observations, leading to a significant set of convenient observations in various directions and depths. Statistical or global analyses require individual and reliable data to be processed in order to determine an independent overall view of the spatial 3-D distribution of different species, after which, the comparison of results could allow the determination of possible spatial abundance correlations. In this work, we use the larger databases at our disposal and better distances as derived from Hipparcos parallaxes. From the comparison with previous results, we conclude that our analysis can be seen as complementary of preceding works. The inversion technique we used gives an average model and not a detailed analysis in one particular direction or at a given distance i.e. the result is a global solution which does not reproduce exactly the initial data. Differences between input data and output values obtained from the model give us information about local anomalies such as errors or differences in column densities $N$ or densities $\rho$, and distances. Obviously, the solution is not unique, but it is the most probable global result in an interval of smoothing length $\xi$, according to the fixed level of confidence of the $\chi^{2}$ criteria.

The shape and extension of the individual clouds and cloud complexes depends on the number of data points considered and on the distribution of target stars in space. An ideal situation will be to have a regular distribution of stars in distance and angle, but in the galaxy, stars are distributed neither uniformly nor randomly, and, in addition, not all the nearby stars were observed for IS purposes. In addition, due to inhomogeneities in the ISM and physical anisotropies, it is hazardous to make claims about reliable $N$-saturated values from an indirect growth curve analysis or by adjusting Voigt profiles, probably because there are necessarily many different LTE temperatures able to describe the ISM.

We summarize some of the results obtained with our inversion technique:

- the IS matter organizes at different scale lengths: annular cloud complexes seem to be drawn for a smoothing length of $60 \mathrm{pc}$, following the structure of the Gould Belt and the annular structure discovered by Egger \& Aschenbach (1995) (see Sect. 5.1);

- dense or quasi-empty regions of $\mathrm{NaI}$ and $\mathrm{HI}$ are qualitatively correlated or anti-correlated (Sect. 5.2);

- several cavities appear between dense regions: the most evident is the LB showing different contours in $\mathrm{NaI}$ and HI, the Loop I and the Loop IV (Sect. 5.1);

- the IS HI is more dispersed than the NaI in the neighbourhood of 300 pc of the Sun (Sect. 5.1); 
- the LB is connected with the galactic halo through "corridors" or "chimneys" of very low density IS matter;

- the NaI and HI clouds we obtained correlate in general with well-known molecular clouds;

- the $\rho_{\mathrm{NaI}} / \rho_{\mathrm{HI}}$ column density ratios vary from one direction to another for low densities. On the contrary, for high density values, the correlation is rather good and the relation between $\rho_{\mathrm{HI}}$ and $\rho_{\mathrm{NaI}}$ is given by $\rho_{\mathrm{HI}} / \rho_{\mathrm{NaI}}<910^{8}$ (Sect. 5.2).

The shape of the Local Bubble appears to be determined from the local cloud complexes in the vicinity of the Sun at the scale given by the smoothing length of $40 \mathrm{pc}(\mathrm{NaI})$. A poorly-defined region is seen in the direction next to the galactic poles. Some probable "tubes", "corridors" or "chimneys", in oblique directions in relation to the galactic plane, could be suggested by the plots. They are the result of a large decay of the density values in these directions. Between $315^{\circ}<l<345^{\circ}$, the disposition of nearby cloud complexes defines another bubble that can be identified as being the Loop I. Unfortunately, we cannot get insight into the interaction region between the Local Bubble and Loop I, because the inversion algorithm has smeared the details through the smoothing length $\xi$.

We have confirmed that the ISM is not uniformly distributed in space: matter concentrates in local places, leaving "tunnels" relatively empty of matter. On the other hand, matter appears to be distributed in shells or sheets, with scales from a pc to a hundredth of pc. Each species and ionisation degree could eventually be distributed in space in different ways to that imagined before and could be representative of different mean temperatures and densities, due to matter inhomogeneities and physical anisotro- pies. The crude temperature qualification as cold, warm and hot IS media is not very useful when interfaces between all these media will be probably the common rule.

Our aim is to pursue the analysis of other IS absorbers (forthcoming works) to determine their spatial distribution independently of each other and then to search for their spatial, qualitative and quantitative correlations, and relations with stellar associations or groups.

Acknowledgements. We thank R. Lallement for useful discussions and C. Pichon for a critical reading of the manuscript. We also thank E. Chereul for his computer skills with Figs. 7 and 8 .

\section{Appendix A: Implementation of the inverse method}

Recall that the column density, $N$, is given as a function of $\alpha$ by:

$$
\begin{aligned}
N & =\int_{0}^{R} \rho(r) \mathrm{d} r \\
& =\int_{0}^{R} \rho_{0} \exp (\alpha(r)) \exp \left(-\frac{|r \sin (b)|}{h_{0}}\right) \mathrm{d} r \\
& :=g(\alpha) .
\end{aligned}
$$

The determination of the density from column densities is an ill-posed problem. Solving such a problem requires regularization techniques (Craig \& Brown 1986), such as Bayesian techniques (Tarantola \& Valette 1982). In the method implemented, we assume an a priori knowledge of the 3D density field (the null information). The conditional probability density $f_{\text {post }}(\alpha \mid N)$ for the vector $\alpha$ of the unknown model parameter (describing the full 3D density field), given the observed data $N$ (the density column along the $\mathrm{n}$ lines of sight) obeys Bayes' theorem:

$f_{\text {post }}(\alpha \mid N)=\mathcal{L}(N \mid \alpha) f_{\text {prior }}(\alpha)$,

where $\mathcal{L}$ is the likelihood function and $f_{\text {prior }}$ stands for the a priori density function for the parameter vector. If we assume that both the a priori probability and the errors in the data are distributed as Gaussian functions, we can write:

$$
\begin{aligned}
f_{\text {post }}(\alpha \mid N)= & A \exp \left(-\frac{1}{2}(N-g(\alpha))^{T} \cdot C_{\mathrm{d}}^{-1} \cdot(N-g(\alpha))\right. \\
& \left.-\frac{1}{2}\left(\alpha-\alpha_{0}\right)^{T} \cdot C_{0}^{-1} \cdot\left(\alpha-\alpha_{0}\right)\right)
\end{aligned}
$$

with, respectively, the variance-covariance matrix $C_{\mathrm{d}}$ and $C_{0}$ of the observed data and the a priori parameter. $\alpha_{0}$ is the a priori value of the parameter and $A$ is a normalisation constant. $T$ refers to the transposed matrix.

The minimum of the argument of the exponential in (A) is shown (by a simple variational argument; Tarantola \& Valette 1982) to obey the implicit equation:

$$
\begin{aligned}
\tilde{\alpha}= & \rho_{0}+C_{0} \cdot G^{*} \cdot\left(C_{\mathrm{d}}+G \cdot C_{0} \cdot G^{*}\right)^{-1} . \\
& \left(D+G \cdot\left(\tilde{\alpha}-\alpha_{0}\right)-g(\tilde{\alpha})\right),
\end{aligned}
$$

with $G_{k}$, the matrix of partial derivatives:

$G=\left(\frac{\partial g}{\partial \alpha}\right)$

Explicitly, $G$ is a following linear operator, whose kernel is:

$\rho_{0} \exp (\alpha(r)) \exp \left(-\frac{|r \sin (b)|}{h_{0}}\right)$.

This minimum is found using an iterative procedure:

$$
\begin{aligned}
\tilde{\alpha}_{[k+1]}= & \alpha_{0}+C_{0} \cdot G_{[k]}^{*} \cdot\left(C_{d}+G_{[k]} \cdot C_{0} \cdot G_{[k]}^{*}\right)^{-1} \\
& \left(N+G_{[k]} \cdot\left(\tilde{\alpha}_{[k]}-\rho_{0}\right)-g\left(\tilde{\alpha}_{[k]}\right)\right)
\end{aligned}
$$

where the subscript $[k]$ refers to the iteration order and $G^{*}$ the adjoint operator of $G$. In this scheme the minimum corresponds to $\tilde{\alpha}=\alpha_{[\infty]}$ and, in practice, is found when the algorithm converges (about 10 iterations). In the first step, the determination of $\tilde{\alpha}$ is done along each line-ofsight where there is data.

The matrix $S_{[k]}=G_{[k]} \cdot C_{0} \cdot G_{[k]}^{*}$ is computed as follows :

$$
\begin{aligned}
\left(S_{[k]}\right)_{i, j}= & \int_{0}^{R_{i}} \int_{0}^{R_{j}} \rho_{0}^{2} \exp \left(\alpha_{[k]}(r)+\alpha_{[k]}\left(r^{\prime}\right)\right) \\
& \times \exp \left(-\frac{\left(\left|r \sin \left(b_{i}\right)\right|+\left|r^{\prime} \sin \left(b_{j}\right)\right|\right)}{h_{0}}\right) C_{0}\left(r, r^{\prime}\right) \mathrm{d} r \mathrm{~d} r^{\prime},
\end{aligned}
$$


with:

$C_{0}\left(r, r^{\prime}\right)=\sigma_{\alpha}^{2} \exp \left(-\frac{r^{2}+r^{\prime 2}-2 r r^{\prime} \cos (\theta)}{\xi^{2}}\right) ;$

$\theta$ is the angle between the two lines-of-sight where there are data $\left(N_{i}, N_{j}\right)$.

The vector $W_{i}=\left(N+G_{[k]} \cdot\left(\tilde{\alpha}_{[k]}-\alpha_{0}\right)-g(\tilde{\alpha})_{[k]}\right)_{i}$ is explicitly computed:

$$
\begin{aligned}
W_{i}= & N_{i}+\int_{0}^{R_{i}}\left(\alpha_{[k]}(r)-1\right) \rho_{0} \\
& \times \exp \left(\alpha_{[k]}(r)\right) \exp \left(-\frac{\left|r \sin \left(b_{i}\right)\right|}{h_{0}}\right) \mathrm{d} r
\end{aligned}
$$

(when the value of the prior $\alpha_{0}=0$ ).

At each step, the linear system is solved using a Cholesky decomposition (Press et al. 1990). Then, after reaching the minimum, it is possible to extrapolate $\tilde{\alpha}$ at each point in space:

$$
\begin{aligned}
\tilde{\alpha}(r)= & \sum_{i}\left(S^{-1} W\right)_{i} \int_{0}^{R_{i}} C_{0}\left(r, r^{\prime}\right) \rho_{0} \exp \left(\tilde{\alpha}\left(r^{\prime}\right)\right) \\
& \times \exp \left(-\frac{\left|r^{\prime} \sin \left(b_{i}\right)\right|}{h_{0}}\right) \mathrm{d} r^{\prime}
\end{aligned}
$$

where $\tilde{\alpha}\left(r^{\prime}\right)$ is known and has been computed along each line-of-sight from Eq. (A.5).

More details on the method are given in Valette and Vergely (in preparation) and Vergely (1998).

\section{References}

Bertout, C., Robichon, N., \& Arenou, F. 1999, A\&A, 352, 574 Chen, B., Vergely, J.-L., Valette, B., \& Carraro, G. 1998, A\&A, 336, 137

Cox, D. P., \& Reynolds, J. 1987, ARA\&A, 24, 499

Craig, I. J. D., \& Brown, J. C. 1986, Inverse Problems in Astronomy (Adam Hilger Ltd., Bristol and Boston) de Zeeuw, P. T., Brown, A. G. A., de Bruijne, J. H. J., et al. 1997, in Hipparcos Venice '97 Symposium, ESA SP402, 495

Diplas, A., \& Savage, B. D. 1994, ApJS, 93, 211

Dupin, O., \& Gry, C. 1998, A\&A, 335, 661

Egger, R., \& Aschenbach, B. 1996, MPE Report, 263, 249

Ferlet, R., Vidal-Madjar, A., \& Gry, C. 1985, ApJ, 298, 838

Frisch, P. C. 1995, Space Sci. Rev., 72, 499

Fruscione, A., Hawkins, I., Jelinsky, P., \& Wiercigroch, A. 1994, ApJS, 94, 127

Garcia, B. 1991, A\&AS, 89, 469

Guillout, P., Sterzik, M. F., Schmitt, J. H. M. M., Motch, C., \& Neuhaeuser, R. 1998, A\&A, 337, 113

Gry, C., York, D., \& Vidal-Madjar, A. 1985, ApJ, 296, 593

Gry, C., Lemonon, L., Vidal-Madjar, A., Lemoine, M., \& Ferlet, R. 1995, A\&A, 302, 497

Lallement, R., \& Bertin, P. 1992, A\&A, 266, 479

McKee, C. F., \& Ostriker, J. P. 1977, ApJ, 218, 148

Morton, D. C. 1991, ApJS, 77, 119

Morton, D. C. 1992, ApJS, 81, 883

Neckel, T., Klare, G., \& Sarcander, M. 1980, A\&AS, 42, 251

Osterbrock, D. E. 1989, Astrophysics of gaseous nebulae and active galactic nuclei, University Sciences Books (Oxford University Press)

Perryman, M. A. C., et al. 1997, A\&A, 323, L49

Press, W. H., Teukolsky, S. A., Vetterling, W., \& Flannery, B. P. 1992, Numerical Recipes (Cambridge University Press)

Sfeir, D., Lallement, R., Crifo, F., \& Welsh, B. Y. 1999, A\&A, 346,785

Slavin, J. D. 1989, ApJ, 346, 718

Spitzer, L. 1968, Diffuse Matter in Space (Wiley \& Sons)

Tarantola, A. \& Valette, B. 1982, Rev. Geo. Space Sci, 2, 219

Vallerga, J. V. \& Welsh, B. Y. 1995, ApJ, 444, 702

Vergely, J.-L. 1998, Ph.D. Thesis, Strasbourg Observatory and Louis Pasteur University

Vergely, J.-L., Egret, D., Freire Ferrero, R., Valette, B., \& Köppen, J. 1997, in Hipparcos Venice '97 Symposium, ESA SP-402, 603

Welsh, B. Y. 1991, ApJ, 373, 556

Welsh, B. Y., Craig, N., Vedder, P. W., \& Vallerga, J. V. 1994, ApJ, 437, 638

Welsh, B. Y., Crifo, F., \& Lallement, R. 1998, A\&A, 333, 101

Welsh, B. Y., Vallerga, J. V., \& McDonald, K. 1998, PASP, 110,827 